\title{
A Novel Surveillance System Applied in Civil Airport
}

\author{
Hua Bo Sun ${ }^{1}$, Min Zhu Zhong ${ }^{1}$, Ling Yun Miao ${ }^{1}$, Xin Wang ${ }^{1}$, Hai Meng Zhao ${ }^{2, \text { a }}$ \\ ${ }^{1}$ China Academy of Civil Aviation Science and Technology, Beijing, 100028, P.R.China \\ ${ }^{2}$ Beijing Key Lab of Spatial Information Integration and Its Applications, Peking University, Beijing 100871, P.R.China
}

\begin{abstract}
Conventional security monitoring of civil airport usually uses a fixed camera to acquire images. There are several problems with performance including difficulties introduced in the information transmission, storage, and analysis of the process. Insect compound eyes offer unique advantages for moving target capture and these have attracted the attention of many researchers in recent years. This paper contributes to this research by proposing a new surveillance system applied in civil airport. We discuss the finished bionic structure of the system, the development of the bionic control circuit, and introduce the proposed mathematical model of bionic compound eyes for data acquisition and image mosaic. Image matching for large view is also illustrated with different conditions. This mode and algorithm effectively achieve safety surveillance of airport with large field of view and high real-time processing.
\end{abstract}

\section{Introduction}

Moving target detection has been applied in many applications, such as video surveillance of civil airport, in recent years. Traditional fixed camera systems for surveillance dealt with the acquired images have two main questions, one is that the field of view is limited, and the other is that data storage is too large with the accumulation. During process acquired image, we must transform the three dimensional objects, which are acquired by high resolution sensors, to two dimensional images. Following this task one must then distinguish and locate the targets using signal-processing techniques. Finally we reverse the two-dimensional information to three-dimensions in order to redisplay the actual objects. The precision of detection and the amount of calculation required are positively correlated in such transformations. The higher the precision required the greater the amount of data involved. Consequently this delivers a less than real-time performance. As a result of these tradeoffs many researchers have turned their attention to a new image acquisition and processing mode called bionic compound eyes ${ }^{[1]}$. Tanida ${ }^{[2]}$ et al proposed a bionic compound eyes imaging system called TOMBO (Thin Observation Module by Bound Optics) which adapted micro lens and photo detectors for the acquisition and receiving assembly of light signals. This information is then processed to restructure the targets. Duparre ${ }^{[3]}$ et al proposed and manufactured the AACO (Artificial Apposition Compound Eye Objective $)^{[4]}$. Insect compound eyes have many advantages in moving target detection. They can detect and recognize targets in large areas using minimal information obtained through feature outline.
Insect compound eyes have many advantages in moving target detection. Insect compound eyes have small size, light weight, large viewing fields, and are very sensitive to moving targets. They can detect and recognize targets in large areas using minimal information obtained through feature outline. Insects can maintain highresolution views of their targets while at the same time maintaining information about other targets in the same view. The mechanism of the compound eyes provides a solution to the tradeoffs between viewing field size, resolution, and real-time processing. These are very important problems that must be addressed when developing moving target detection systems. This paper follows on our previous work on moving object detection with bionic compound eyes applied in surveillance of airport $^{[5]}$.

The remainder of this paper is organized as follows. In section 2 we explain our bionic compound eyes mechanism. Section 3 provides a full overview of our system composition, biometric structure, control system, and a mathematical formulation of the data acquisition and processing model. In section 4 a report on some experimental analysis is discussed. Section 5 closes the paper with some conclusions and directions for future research.

\section{Bionic mechanism}

Compound eyes have many advantages including a large field of view and high sensitivity for moving target detection. Reichardt ${ }^{[6]}$ et al proposed that there are two independent channels in compound eyes. One is called the "large field system" and the other is called the "small field system". The large field system detects global

\footnotetext{
a Corresponding author: sunhb@castc.org.cn
} 
motion and the small field detects moving targets accurately. Our proposed detection algorithm with bionic compound eyes includes two phases. During the first phase we simulate the insect's compound eyes to detect the moving object outline and extract object in the surrounding area. In the second phase of algorithm we simulate the insect's compound eye's mechanism to accurately extract the moving object.

Compound eyes, with large view field, are very sensitive to moving objects region and location and can detect moving objects quickly with little outline information even when these objects are located in large open areas. Contour detection and variable image resolution are probably the two most important characteristics of compound eyes. Based on these characteristics we shall now propose our bionic eyes mechanism which is shown in Fig. 1.

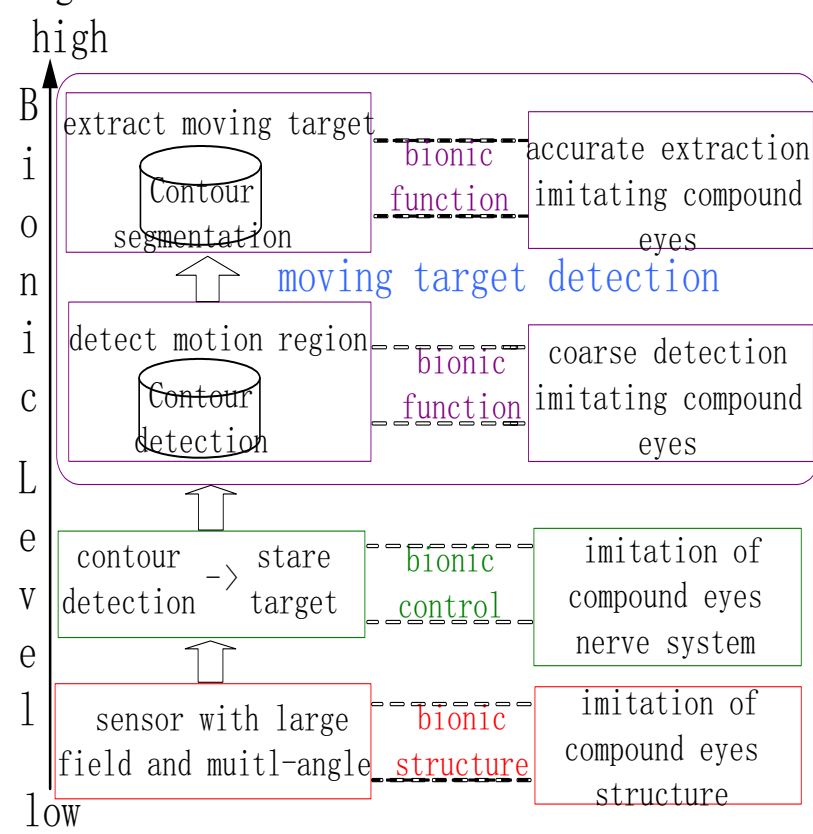

Figure 1. Schematic diagram of our bionic eyes mechanism.

Our bionic compound eyes mechanism, shown in Fig. 1, includes three components: bionic structure, bionic control, and bionic function. The biometric structure is the foundation component which acquires data using the sensors. The bionic control component then performs the contour extraction. The bionic function performs the detection of moving targets. The region of motion is computed by the bionic function. The final component of our bionic eyes mechanism is executed after contour segmentation of the remotely sensed images. The moving target is then extracted. It is clear from the structure of Fig. 1 that biometric level grows from low-level complexity to the highest level of complexity when the moving target is extracted. As commented by Corcoran et al segmentation presents a difficult problem in all object identification methodologies. Consequently segmentation is at the top of the complexity level for the biometrics in Fig. 1. In the next section we will describe, in detail, the bionic compound eyes detection system components.

\section{Bionic surveillance system}

In this section we describe, in detail, the bionic compound eyes detection system. The composition of the system is described in section 3.1. The biometric structure, including details of the cameras used, is described in section 3.2. Section 3.3 provides detailed information on the hardware control system. The final two sections provide the details about the data acquisition and object detection model.

\subsection{Composition of the System}

Based on the mechanism of insect compound eyes for detecting moving targets, we have constructed a bionic compound eyes detection system. The compound eyes structure consists of the large field of view, a multi-angle mechanical structure, a hardware control system, a data acquisition device, and an object detection model. The "electrics" component illustrates the hardware used with electrical components for control, image sensing, processing, and data storage. The mechanical components of the system are the physical components making up the compound eyes structure.

\subsection{Biometric structure}

According to the physical structure of insect compound eyes the mechanical structure of the bionic compound eyes is constructed using high and low resolution cameras. Our compound eyes device, which is shown in Fig. 2, consists of five cameras. Four cameras (all low resolution) surround one camera (central camera - high resolution) to form an equilateral hexagon. The fixed cameras devices are soft connected with the middle mechanical device. When detecting moving targets low resolution cameras detect the presence of moving objects while high resolution camera is responsible for target imaging. Using a servo system to control the angle of the cameras we can control the cameras to not only point to a specific position but also to monitor large areas in several directions.

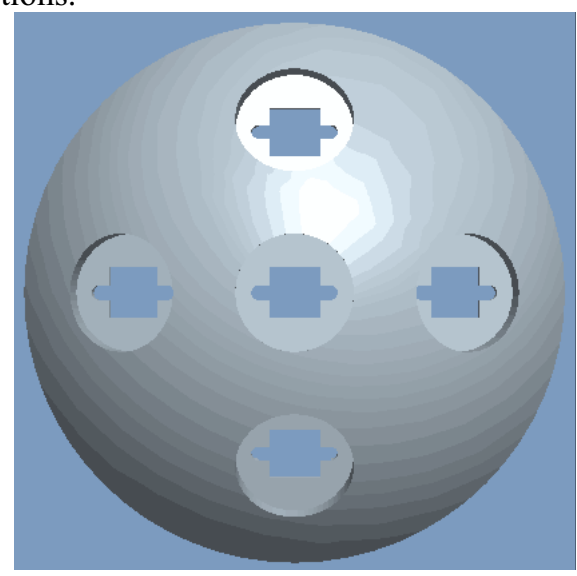




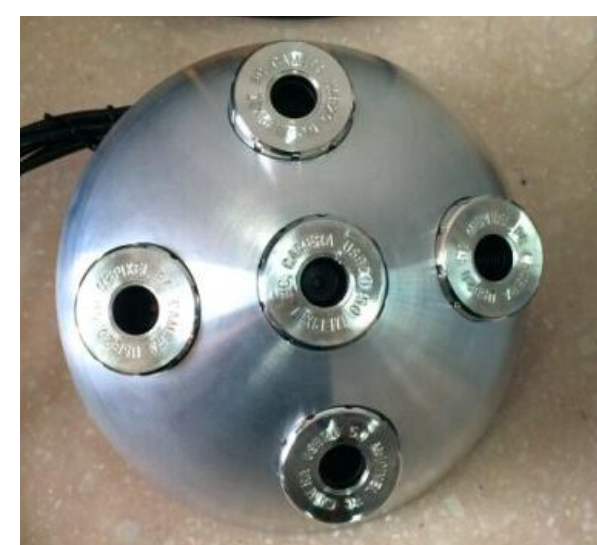

Figure 2. The physical structure of the five cameras on the compound eyes device.

As our system has a relatively large field of view the target will not quickly move out of visual range even if it is moving fast. Many authors agree with this approach such as Nanzhi et al ${ }^{[7]}$ who comment that this also greatly enhances the robustness of any moving target detection systems. Subsequently this increases the detection performance.

\subsection{Control System}

In this section we describe the control system. The bionic compound eye's synchronous capture hardware control system consists of four parts: the DSP (Digital Signal Processor), main control unit, FPGA (FieldProgrammable Gate Array) logic control unit, output unit, and the compound eyes device, physical structure is shown in Fig. 3.

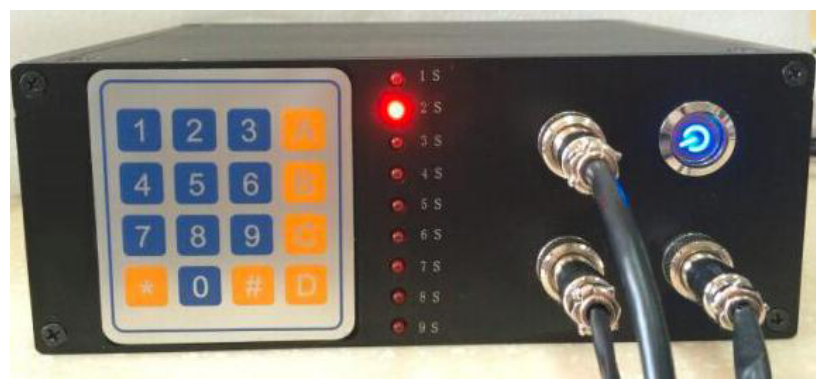

Figure 3. Physical structure of control system.

The workflow of the control system is described as follows: The DSP main control core unit produces multichannel control signals according to the combination of address bus and data bus. These signals are then transported to the FPGA controlling information source for the system. The FPGA generates synchronous sequential control units according to the combinational and sequential logic circuits. As the internal logic unit in FPGA has a precise synchronization trigger function it can generate proper multi-sync pulses whose synchronization error can be controlled in microseconds. In this way the system can fully satisfy the control function requirements. The synchronous pulses from the FPGA, after amplification, are entered into the drive output unit to drive all the shutter control circuits of the compound eyes directly.
The specific details of this are as follows: The shoot interval time can be achieved from counting the input clock and satisfying the parameters. The required waveform can be obtained by reversed phase when the count reaches the predefined value. The entire compound eyes system contains several timing controls. Three-input encoding circuits are needed at least to accomplish eight control modes with a DIP (Dual In-line Package) switch on the hardware circuit. Shoot intervals of eight different simultaneous control modes can be generated by the DIP switch while the modes can be presented by eight different display circuits.

\subsection{Data acquisition model of compound eyes}

Compound eyes system has the advantage that several targets within a given range can be captured simultaneously using multiple sub-eyes. However these sub-eyes can introduce many redundant images. This potential disadvantage can be minimized by re-using these redundant images to assist in reducing the random error in the targets' information.

Pengq ${ }^{[8]}$ proposed a preliminary algorithm for moving targets detection using bionic compound eyes. On the basis of this algorithm we have developed an improvement to his algorithm by proposing a bi-level contour detection model with variable resolution bionic compound eyes, as is shown in Fig. 4.

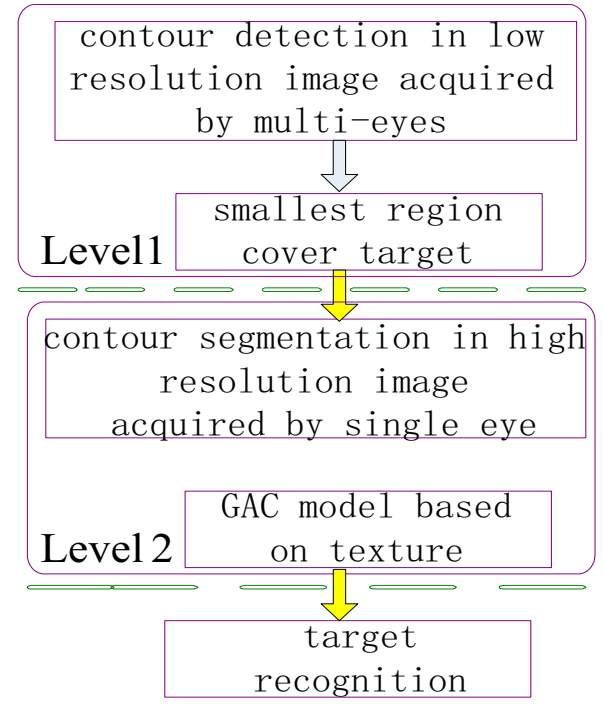

Figure 4. Flowchart diagram of the bi-level contour detection model used in our algorithm.

There are three important components of this algorithm. Firstly there is contour detection which is applied to the lowest resolution images. When this is complete the texture gradient computation is performed by conducting contour segmentation on the high resolution image(s) acquired by the single eye. Finally a Geodesic Active Contour model for texture boundary detection is applied from which the moving target is recognized.

\section{Experimental and result}


In this section we show an example of large view using bionic compound eyes. The example below shows the result processing the captured images using the approach SURF (Speeded-Up Robust Features) feature extraction. In this method, first of all, pre-classify the images according to the image geometric moment and the number of corner points, in order to achieve the automatic selection of image registration algorithm on the basis of the image features. SURF algorithm includes two parts: the feature detection and characterization. Feature characterization is described as

$$
\boldsymbol{v}=\left(\sum d_{x}, \sum d_{y}, \sum\left|d_{x}\right|, \sum\left|d_{y}\right|\right)
$$

Wherer $d_{x}$ is Haar wavelet response in the horizontal direction, $d_{y}$ is Haar wavelet response in the vertical direction. Feature detection is completed by the formula (2) and (3).

$$
\begin{gathered}
I_{\Sigma}(x)=\sum_{i=0}^{i \leq x} \sum_{j=0}^{j \leq y} I(i, j) \\
\boldsymbol{H}(\boldsymbol{x}, \sigma)=\left[\begin{array}{ll}
L_{x x}(\boldsymbol{x}, \sigma) & L_{x y}(\boldsymbol{x}, \sigma) \\
L_{x y}(\boldsymbol{x}, \sigma) & L_{y y}(\boldsymbol{x}, \sigma)
\end{array}\right]
\end{gathered}
$$

$I_{\Sigma}(x)$ stands for the sum of all the pixel gray values and $H_{(X, \sigma)}$ represents Hessian matrix.

The captured images are processed in the order of Fig.5.F and the result is shown as Fig.6.

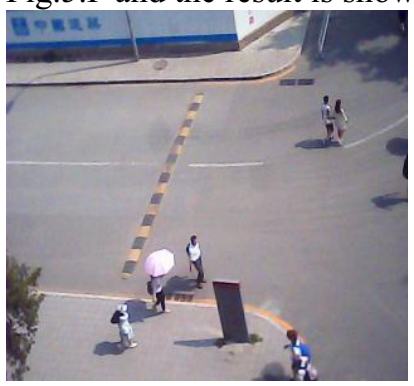

A

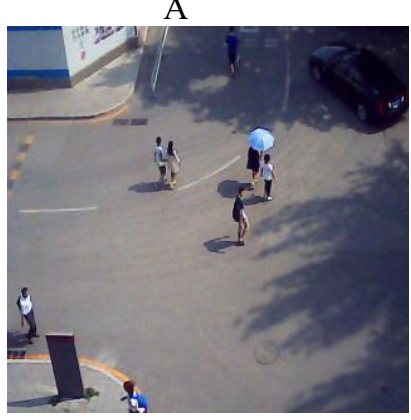

C

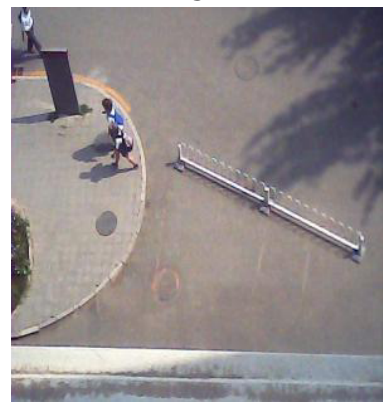

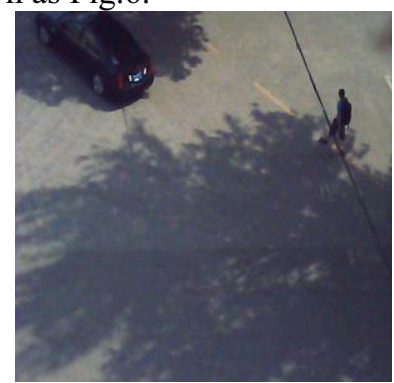

B

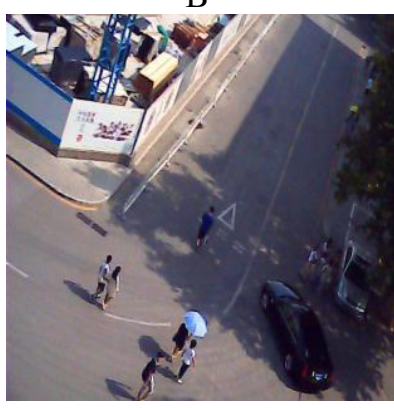

$\mathrm{D}$

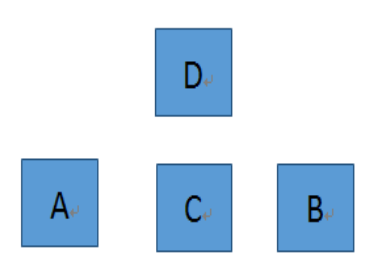

Figure 5. The experimental data captured by Bionic surveillance system.

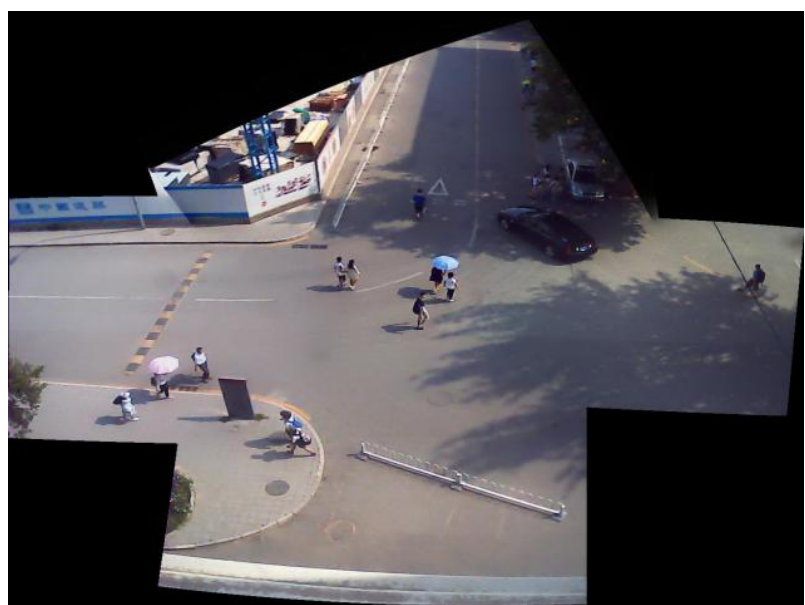

Figure 6. The result of experimental data.

The system we have described, in this paper, for surveillance using bionic compound eyes with almost $180^{\circ}$ field of view. This system achieves a very satisfactory tradeoff between the conflicting objects of: controlling the size of the field of view, the resolution of images captured, and the requirement to perform processing in real-time.

\section{Acknowledgment}

The work presented in this paper is supported by The Scholarship for International Young Scientists of NSFC (National Natural Science Foundation of China) (ID: U1333124 \& U1433102). We thank the editor and reviewer for their contributions to improvements to this paper.

\section{References}

1. Paragious N, Deirche R. Geodesic Active Contours and Level Sets for the Detection and Tracking of Moving Objects. IEEE Transactions on Pattern Analysis and Machine Intelligence, 22, 266280(2000).

2. Yamada K, Ishida S, Shougenji R, Tanida J. Development of three dimensional endoscope by Thin Observation by Bound Optics(TOMBO). Automation Congress, 199-205(2006).

3. Duparré J, Wippermann F, Dannberg P, Bräuer A. Artificial compound eye zoom camera. Bioinspiration biomimetics, 3, 1806-1823(2008).

4. Duparre J, Wippermann F, Darnnberg P, Schreiber P, Bräuer A, Völkel R, Scharf T. Micro-optical artificial compound eyes-from design to experimental verification of two different concepts. SPIE, 5962, 112(2005).

5. Huabo Sun, Haimeng Zhao and Peter Mooney et al, A Novel System for Moving Object Detection Using 
Bionic Compound Eyes, Journal of Bionic Engineering, 8,313-322(2011).

6. Reichardt W. Evaluation of optical motion information by movement detectors. Journal of Comparative Physiology A: Neuroethology, Sensory, Neural, and Behavioral Physiology, 161, 533547(1987).

7. Nanzhi Jiang, Renbiao Wu, Jian Li. Super resolution feature extraction of moving targets. IEEE Transactions on Aerospace and Electronic Systems, 37, 781-793(2001).

8. Pengqi Gao. Research on moving target detection using bionic compound eyes on UAV. Dissertation of Peking University, 21-26(2009). 